\title{
FTIR INVESTIGATIONS OF RHODOPSIN AND RHODOPSIN MUTANTS IN DETERGENT AND RECONSTITUTED INTO MEMBRANES
}

M. BECKa ${ }^{a}$ T.P. SAKMAR ${ }^{b}$ and F. SIEBERT ${ }^{a}$

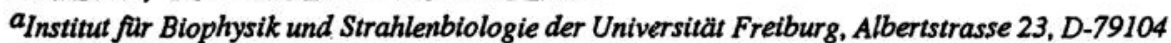

Freiburg. Germany

b Howard Hughes Medical Institue, Rockefeller University, 1230 York Ave, New York, N.Y. 10021, U.S.A.

The photoreceptor rhodopsin in rod cells is member of the superfamily of seven transmembrane helix receptors that activate G-proteins. The chromophore 11-cis-retinal is covalently bound to lysine -296 by a protonated Schiff base linkage. Light-absorption induces isomerization of the chromophore to the all-trans form and through a series of intermediates, metarhodopsin II (MII) is formed. MII is the active conformation of rhodopsin that binds the visual G-protein transducin and thereby triggers the visual transduction cascade.

MII exists in an equlibrium with the preceeding intermediate metarhodopsin I (MI). Under certain conditions it is possible to stabilize the MI-state. These conditions include low temperature $\left(<-20^{\circ} \mathrm{C}\right)$ and increased $\mathrm{pH}(>8)$. Furthermore it seems that the lipidbilayer of the membrane influences the equilibrium, because detergent-solubilized rhodopsin strongly favours the MII-conformation, by destabilizing the MI conformation (1). Therefore, it appears interesting to investigate the influence of the detergent on the structure of MI.

We have applied FT-IR difference spectroscopy on native bovine rhodopsin and rhodopsin mutants expressed in COS cells both solubilized in dodecyl maltoside and reconstituted into lipids. Rhodopsin versus MI- and rhodopsin versus MII-photoproduct FT-IR difference spectra were recorded in order to study conformational changes and environmental changes of specific groups within the protein.

The rhodopsin-MII difference spectra of lipid-reconstituted rhodopsin and detergentsolubilized rhodopsin show no large differences. Only a difference band at $1734(-)$ ! $1745(+) \mathrm{cm}^{-1}$ which is present in the spectrum of the solubilized sample and could be assigned to the internal carboxyl group Glu122 is not present in the spectrum of membrane bound rhodopsin. Thus, the detergent influences somewhat the structure of MII. Comparison of the rhodopsin-MI difference spectra also show high similarities in band positions and relative intensities. The main chromophore absorption bands are observed between $1100 \mathrm{~cm}^{-1}$ and $1300 \mathrm{~cm}^{-1}$ while the frequency range $1300 \mathrm{~cm}^{-1}-1500$ $\mathrm{cm}^{-1}$ covers many protein vibrations for which chemical assignments are not yet available. The close correspondence between the spectra indicates that irrespective of the preparation, the essential conformational changes upon photoconversion to MI are the same. A characteristic broad band with a maxiumum at $1701 \mathrm{~cm}^{-1} \mathrm{can}$ be observed in the spectrum of the lipid-reconstituted protein as well as in ROS-rhodopsin. This structure is missing in the spectrum of solubilized rhodopsin. It has been suggested that this band is due to a protonation of the counterion Glu113 upon MI formation (2). Concomitantly,

173

J. C. Merlin et al. (eds.), Spectroscopy of Biological Molecules, 173-174.

(1) 1995 Kluwer Academic Publishers. Printed in the Netherlands. 
another carboxyl group that later was identified as Glu122 (3) would then be deprotonated causing a negative band at $1734 \mathrm{~cm}^{-1}$. But a shift of the band of protonated Glu122 from 1734 to $1700 \mathrm{~cm}^{-1}$ could not be excluded. In any case, both molecular events would be reverted with the formation of MII. Thus, the observation that the characteristic band at $1700 \mathrm{~cm}^{-1}$ is missing in MI solubilized in detergent signifies that some aspects of MII have already been adopted in MI, and this could explain the destabilizing effect of the detergent on MI. Corresponding measurements on rhodopsin mutants will further clarify these processes.

It is known that replacement of histidine 211 by phenylalanine changes the MI/MII equilibrium. Therefore, we are interested in the influence which is exerted by replacing this residue on the structures of rhodopsin, MI and MII. The infrared difference spectra show that bands caused by Glu122 are strongly affected in rhodopsin, MI and MII. Chromophore bands and most of the other protein bands are not influenced. This shows that there must be either a direct interaction of His211 with Glu122, or that the replacement causes an indirect alteration. Since otherwise the mutation causes only small effects on the protein structure, a more direct effect appears more plausible. Such an interaction would not contradict the helical wheel model derived by Baldwin (4). However, no clear interpretation can be given for the effect the mutation has on the MI/MII equilibrium.

\section{References}

1. König, B., Welte, W., Hofman, K.P. (1989), FEBS Lett. 257, 163-166.

2. Ganter, U. M., Schmid, E.D., Perez-Sala, D., Rando, R.R., Siebert, F. (1989), Biochemistry 28, 5954-5962.

3. Fahmy, K., Jäger, F., Beck, M., Zvyaga, T.A., Sakmar, T.P. (1993), Proc. Natl. Acad. Sci. USA 90, 10206-10210..

4. Baldwin, J.M. (1993) EMBO J. 12, 1693-1703. 